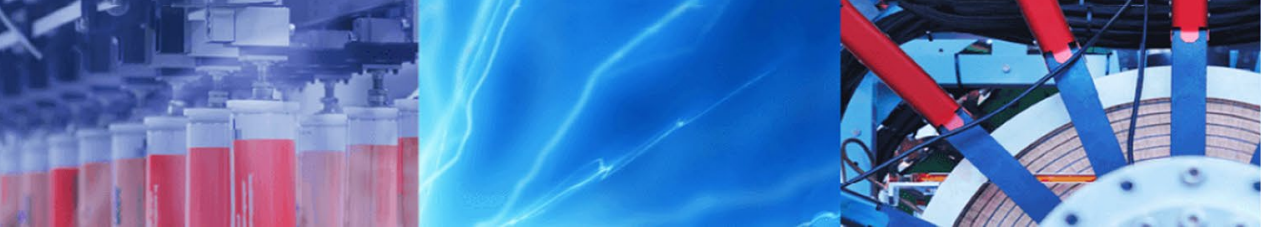

Research Article

\title{
Experimental realization of electromagnetic toroidal excitation for microwave applications
}

\author{
V. P. Sarin ${ }^{1} \cdot$ P. V. Vinesh ${ }^{1} \cdot$ Mani Manoj $^{2} \cdot$ Aanandan Chandroth $^{2} \cdot$ Mohanan Pezholil $^{2} \cdot$ Vasudevan Kesavath $^{2}$
}

(c) Springer Nature Switzerland AG 2019

\begin{abstract}
An experimental realization of resonant toroidal dipole excitation in the microwave regime is presented in this paper. The metasurface proposed herewith is an asymmetric double split-ring resonator exhibiting Fano-like resonance profile. One could observe that cascading the metasurface results in near-field transverse and longitudinal coupling creating dual-band toroidal moments on the structure. This planar scheme will simulate the development of enhanced light-matter interaction, electromagnetic wave slowdown and sensor design. Here we report the experimental realization of the phenomenon in the S-band frequencies, and the results are verified using multipole scattering analysis.
\end{abstract}

Keywords Asymmetric metasurface $\cdot$ Fano resonance $\cdot$ Toroidal metamaterials $\cdot$ Toroidal dipoles

\section{Introduction}

Recent research interest has been focused on the scattering studies of a special type of excitation known as toroidal dipoles in composite metamaterial structures. The classical electromagnetic theory accounts only the scattering from electric and magnetic dipoles in the multipole scattering formalism [1]. An electric dipole is created by the separation of positive and negative charges, whereas a magnetic dipole is generated by virtue of circulating currents on a composite. The simultaneous excitation of electric and magnetic dipoles is used to tailor both magnitude and phase of transmission/reflection coefficients from a metamaterial composite [2, 3]. Toroidal dipoles are exempted from classical multipole expansion theory and are caused due to the surface current circulating on a metallic torus along its meridians. Their excitation in the nuclear system was first reported by Zel'dovich [4]. The farfield scattering pattern of a toroidal dipole is indistinguishable from that of an electric dipole [5]. Recently, there has been an immense interest in the development of toroidal metamaterials for electromagnetic wave manipulations. Artificial media exhibiting strong toroidal excitation are used for the creation of electromagnetically induced resonant transparency $[6,7]$. They can also be used for creating a negative refractive index medium [8]. In conventional artificial media, a careful design procedure must be followed to suppress the excitation of electric and magnetic dipoles and to enhance toroidal dipole response. One such 3D configuration is the split loop structures immersed in a dielectric medium [9]. Singh et al. used asymmetric mirrored double split-ring structure and a combination of the split ring with dogbone metalization to generate toroidal dipole resonances in planar media $[10,11]$. A different configuration proposed by the same group achieves Fano resonance observed in electromagnetically induced transparency system in addition to the toroidal response [12]. Recently, toroidal metamaterials have been successfully used for sensing and switching applications [13, 14]. Both Fano resonance and toroidal response can also be simultaneously excited in a stacked configuration of asymmetric split-ring resonators [15]. Localized toroidal spoof

\footnotetext{
$\triangle$ V. P. Sarin, sarincrema@gmail.com | 'Department of Electronics, Government College Chittur, Palakkad, Kerala 678104, India. ${ }^{2}$ Department of Electronics, Centre for Research in Electromagnetics and Antennas, Cochin University of Science and Technology, Cochin, Kerala 682022, India.
} 
plasmon polaritons are observed in split-ring resonators arranged in cylindrical fashion at microwave frequencies [16]. All dielectric metamaterials also support toroidal dipole excitation [17]. Dong et. al performed a variety of studies on toroidal dipole excitation in the microwave and $\mathrm{THz}$ regime [18-20]. Electromagnetic toroidal excitations in nature and artificial metamaterials are well reviewed in the literature [21]. An interesting application of toroidal metamaterials is the ease of creation of electromagnetic cloaks. It is worth mentioning that simultaneous excitation of electric and toroidal resonances could reduce the scattering cross section of the target [22-24]. The combination of both toroidal and dipole resonances is popularly known as an anapole. An anapole resonance is characterized by a strong electromagnetic energy concentration in the near field, and hence, it can be used for energy localization $[25,26]$. Recently, toroidal resonators composed of water have been effectively used for achieving electromagnetic invisibility [27]. It is interesting to observe that dual-band toroidal response could be achieved by using a single array metasurface composed of trimmer clusters of high index dielectric cylinders [28]. It focuses on inter-cluster and intra-cluster coupling between neighboring particles to achieve dual-band response.

In this paper, the authors propose the use of a novel metamaterial for realizing resonant toroidal modes in the microwave regime. The advantage of the proposed design is that only two identical cascaded layers are only required to excite toroidal dipole modes. Cascading the metasurface enables both inter-layer and intra-layer near-field coupling which creates dual-band toroidal dipole moments on the structure for normal incidence. The advantage is that the excited toroidal moment significantly improves magnetic field confinement for the Fano resonance resulting in high quality factor for the Fano resonant peak. Simulation studies used for the characterization of metamaterial have been performed using CST Microwave Studio platform, and experiments are conducted inside and anechoic chamber using PNA E8362B network analyzer.

\section{Design of the metasurface}

Evolution of the proposed metamaterial structure is shown in Fig. 1. The fundamental structure constitutes a symmetric double split-ring resonator exhibiting strong electric dipole resonance as shown in Fig. 1a. Creation of an asymmetry in the structure by varying the width of vertical right arm as shown in Fig. $1 \mathrm{~b}$ creates a Fano-like resonant peak in the transmission spectrum. Two such asymmetric surfaces are cascaded to get the final metasurface shown in Fig. 1c. The inter-element thickness ' $d_{z}$ ' is optimized after running parametric simulations in CST Microwave Studio. (a)

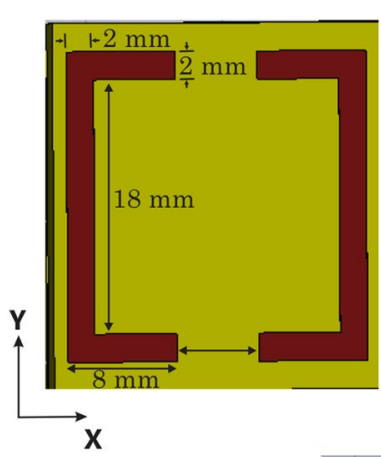

(b)

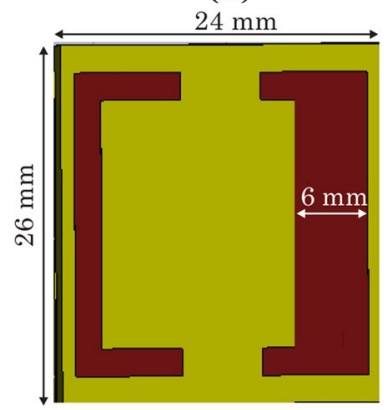

(c)

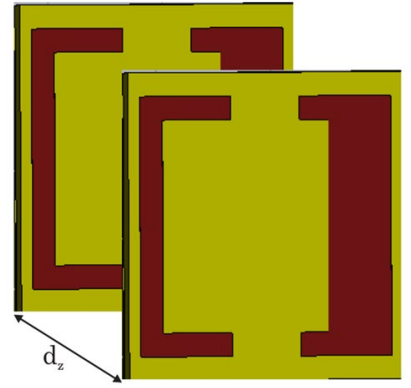

Fig. 1 Evolution of the metamaterial. a Symmetric double split-ring resonator, b asymmetric double split-ring resonator, and $\mathbf{c}$ stacked asymmetric double split-ring resonator

Unit cell parameters of the samples are indicated in the figure. The samples are fabricated using standard photolithographic manufacturing techniques. We used standard epoxy substrate having relative dielectric constant 4.4 and height $1.6 \mathrm{~mm}$ for fabrication. The array has a footprint of $210 \mathrm{~mm} \times 300 \mathrm{~mm}$, and two such arrays are cascaded using Perspex holders to yield the final design. The final fabricated structure is a $8 \times 10$ element array. Thickness of the printed copper metalization is $35 \mu \mathrm{m}$.

\section{Characterization of the metamaterial}

Unit cell simulations have been performed to retrieve the transmission spectra of the arrays for normal incidence. For that, periodic boundaries are applied on the top, bottom, left and right faces of the unit cell and frequency domain simulations have been performed using Floquet port excitation in CST Microwave Studio. Measurements have been performed inside an anechoic chamber using two ultrawideband horn antennas configured in transmission and reception mode. Initially, a THRU calibration is made with antenna separation held at $2 \mathrm{~mm}$. Then the samples are placed in between the antennas in such a way that the electric field of the emitted radiation is made parallel to the metallic strip along $Y$-direction and resultant transmission spectra are measured for normal incidence. 
An overview of the measured and simulated transmission spectra of the initial configuration shown in Fig. 1a is depicted in Fig. 2. The simulation and measurement are showing excellent agreement with each other. The reflective resonance at $3.37 \mathrm{GHz}$ is characterized by a strong contribution from electric dipole excitation, and correspondingly, the transmission coefficient is extremely weak. Computed surface current distribution at resonance is depicted in Fig. 2b. Two symmetric half-wavelength resonant distributions with a current minimum at the open ends and maximum at the center are observed on each plate. This confirms the presence of an electric dipole moment $P_{y}$ excited on the composite.

Transmission measurements are repeated for the asymmetric single layer structure shown in Fig. 1b, and the corresponding transmission spectrum is illustrated in Fig. 3. Introducing an asymmetry in the structure creates a tunnel band centered at $3.23 \mathrm{GHz}$ as shown in Fig. 3a. Near-unity transmission is observed for this resonance. Excitation of electric dipole resonance is characterized by a dip in transmission coefficient at $3.1 \mathrm{GHz}$. The reason behind this newly introduced pass band resonance can be studied by verifying the surface currents excited on the structure as shown in Fig. 3b. As shown, the passband is caused due to the circular loop current distribution excited on the composite. This Fano-like resonance profile is contributed due to the simultaneous excitation of electric and magnetic moments on the structure. The circular

(a)

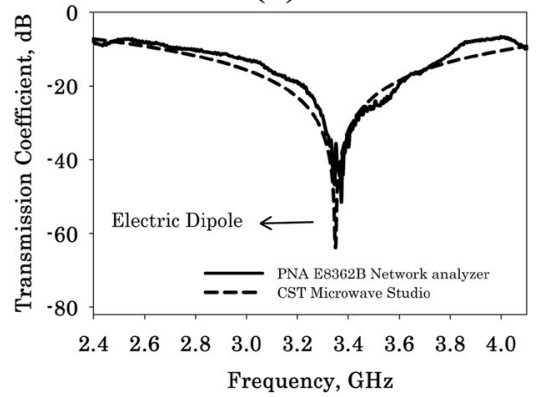

(b)

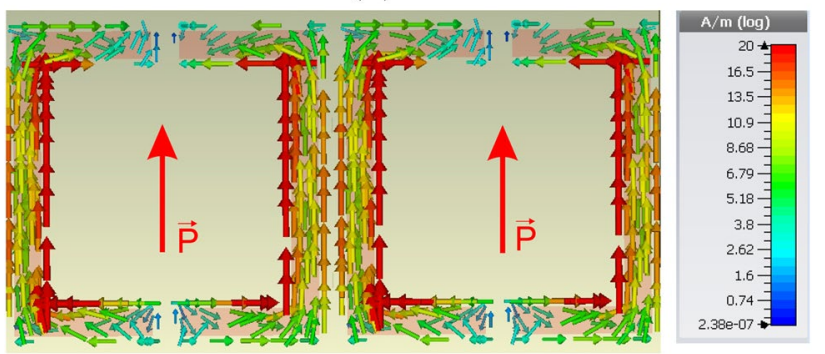

Fig. 2 Characterization of the symmetric unit cell. a Transmission spectrum and $\mathbf{b}$ surface current distribution at resonance (a)

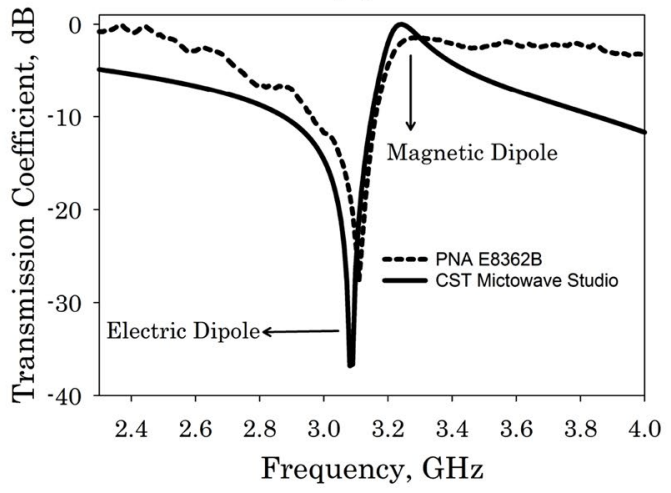

(b)

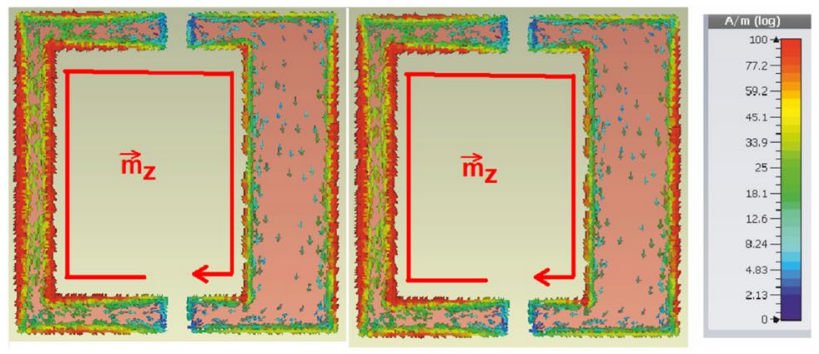

(c)

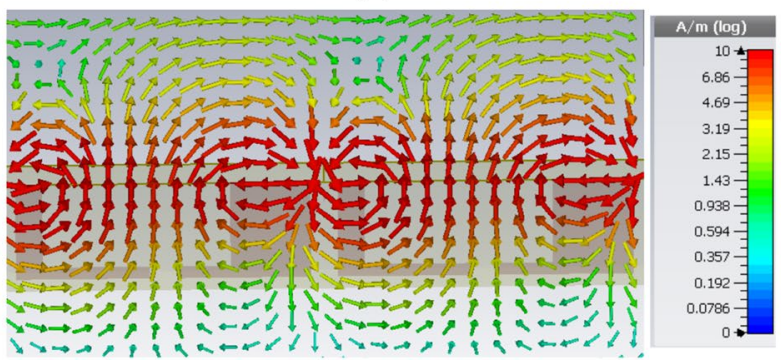

Fig. 3 Characterization of the asymmetric single layer metamaterial. a Transmission coefficient, b simulated surface current distribution at $3.23 \mathrm{GHz}$ and c simulated magnetic field profile at $3.23 \mathrm{GHz}$

loop current excited on the $X Y$ plane of the metasurface creates a magnetic moment $\left(M_{z}\right)$ on the composite. All the elements lying in the plane are excited in phase, and hence, magnetic moments on each loop will be oscillating in phase. Figure $3 c$ illustrates the magnetic field intensity distribution in a plane perpendicular to the array. It is obvious that the resonant magnetic fields at the center of two consecutive elements are in phase. The Q-factor of this resonance is found to be 19 in simulation.

The final stacked metasurface depicted in Fig. $1 \mathrm{c}$ is assembled by fixing Perspex spacers in between the arrays for mechanical support. Figure 4 illustrates the transmission spectra of this stacked asymmetric composite for $d_{z}=4.5 \mathrm{~mm}$. The structure shows two resonances for the band of interest as illustrated in the figure. The first resonance is found to be at $2.6 \mathrm{GHz}$, and the second one is at 


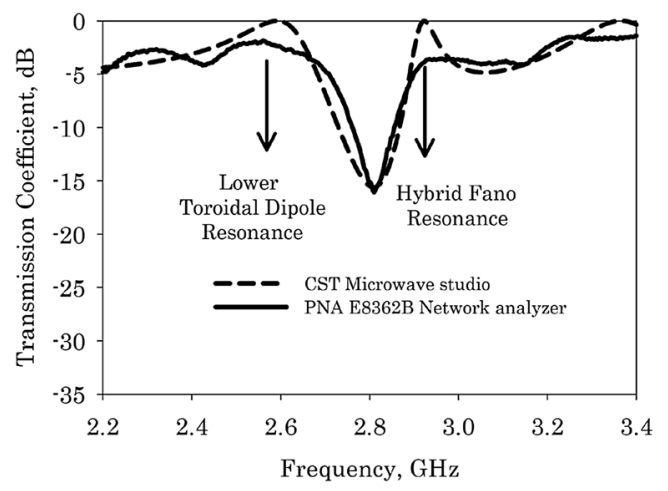

Fig. 4 Transmission coefficients of the stacked asymmetric metasurface

$2.92 \mathrm{GHz}$. The electric dipole resonant dip is found to be at $2.8 \mathrm{GHz}$, and correspondingly, the transmission coefficient is very low of the order of $-16 \mathrm{~dB}$. The slight mismatches between measurement and simulation are due to measurement tolerances. Q-factors of these resonances are found to be 10 and 41 , respectively, in simulation. It is interesting to note that the quality factor for the second resonance is significantly enhanced. The first resonance is identified as the toroidal dipole resonance, and the second one is the hybrid Fano resonance. These conclusions are reached after performing multipole scattering analysis of the unit cell.

\section{Multipolar scattering studies}

Multipole scattering theory has been utilized to study the resonant mechanism of the final structure for $d_{z}=5.5 \mathrm{~mm}$. Radiating power from the induced multipoles can be calculated by integrating the spatially distributed current distribution over the unit cell. The multipole amplitudes can be calculated as [5]

$P=\frac{1}{i \omega} \int J \mathrm{~d}^{3} r$

$M=\frac{1}{i \omega} \int[\vec{r} X j] d^{3} r$

$T=\frac{1}{10 c} \int\left[(\vec{r} \cdot \vec{j}) r-2 r^{2} J\right] d^{3} r$

where $P$ is the electric dipole moment, $M$ is the magnetic dipole moment, $T$ is the toroidal moment, $c$ is the velocity of light in vacuum, $\vec{r}$ is the displacement vector from the origin, $\omega$ is the angular frequency and $J$ is the surface current density retrieved from simulations. The total power (a)

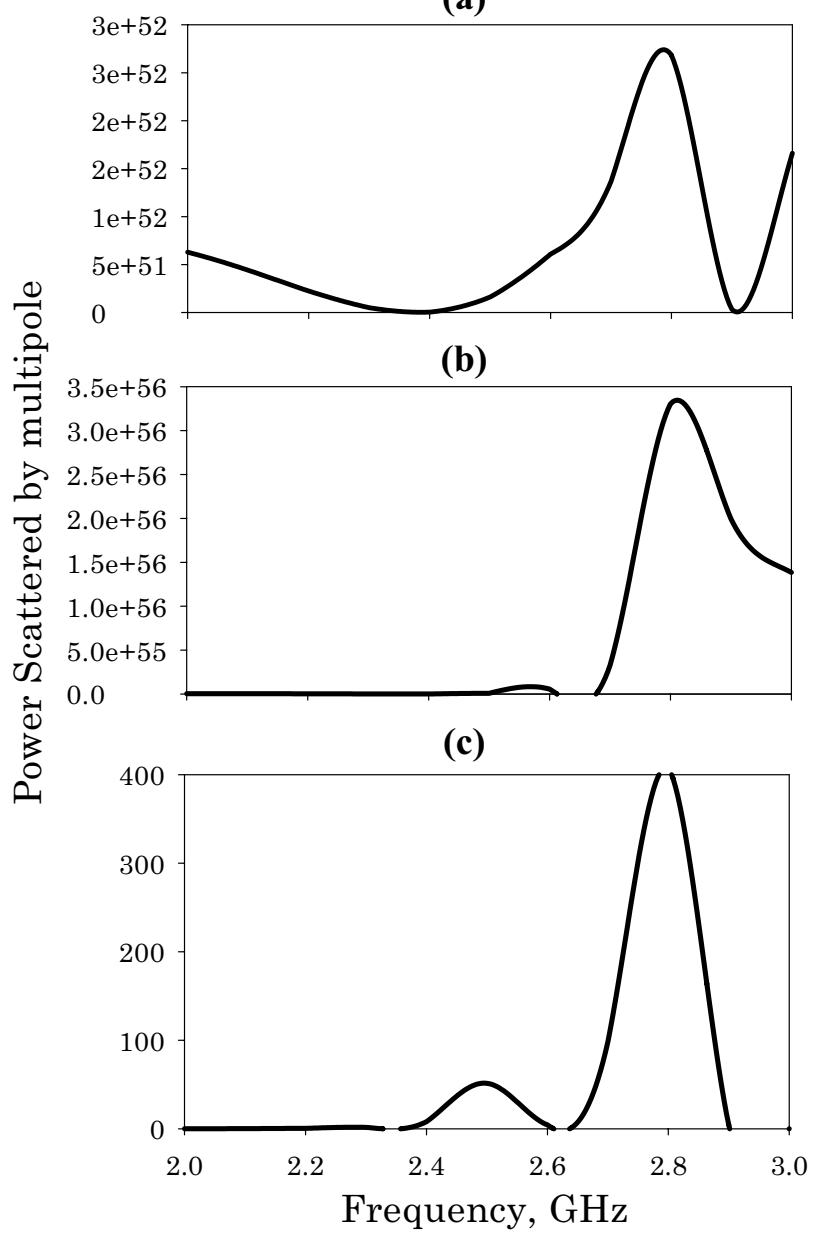

Fig. 5 Power radiated by multipoles. a Power radiated by electric dipole $\left(P_{y}\right)$, b power radiated by magnetic dipole $\left(M_{z}\right)$ and $\mathbf{c}$ power radiated by toroidal dipole $\left(T_{y}\right)$

radiated from different multipole moments can be formulated as

$I=\frac{2 \omega^{4}}{3 c^{3}}|P|^{2}+\frac{2 \omega^{4}}{3 c^{3}}|M|^{2}+\frac{2 \omega^{6}}{3 c^{5}}|T|^{2}+\cdots$

Corresponding scattered power from the electric, magnetic and toroidal moments for the final structure is shown in Fig. 5. It is seen that the electric dipole moment $P_{y}$ has a significant contribution throughout the full range of frequencies. The toroidal dipole $T_{y}$ has a significant contribution for the lower resonance centered at $2.54 \mathrm{GHz}$ in which the power radiated from the electric dipole moment is showing a significant dip. This dip is caused due to the scattering cancelation from anti-symmetric current distribution from stacked elements. Electromagnetic transmission is achieved by the virtue of the toroidal moment $T_{y}$ on the composite. The presence of toroidal moment interferes destructively giving the lower transmission 
peak at this resonance. The second resonance at $2.87 \mathrm{GHz}$ is contributed by the combined effect of electric dipole $P_{y}$, toroidal dipole $T_{y}$ and magnetic dipole $M_{z}$ and hence can be named as the hybrid Fano resonance. The presence of magnetic and toroidal dipoles reduces reflection from the electric dipole moment giving near-unity transmission at this frequency. We have also performed the multipole scattering analysis of the asymmetric single layer structure depicted in Fig. $1 \mathrm{~b}$. It is noted that in that case, the contribution from the toroidal term is absent and resonance is contributed due to the simultaneous excitation of electric and magnetic dipole moments as that observed in Fano resonance-based transparency windows.

In order to verify the presence of toroidal excitation on the structure, the simulated magnetic field distributions in the $X Z$ computational domain for the two resonances are taken into consideration and are shown in Fig. 6 . In the simulation, the wave is propagating along $+Z$ direction and the structure lies in the $X Y$ plane. It is observed that for the lower resonance at $2.54 \mathrm{GHz}$, the magnetic moments excited on two consecutive stacked elements are out of phase and it enables transverse intra-layer coupling. The resulting magnetic vortex formed in between the adjacent asymmetric arms creates toroidal dipole polarization $T_{y}$ on the composite. The surface currents on the stacked plates will be out of phase at this frequency. The toroidal moment $T_{y}$ excited on each cell is oscillating in phase

(a)

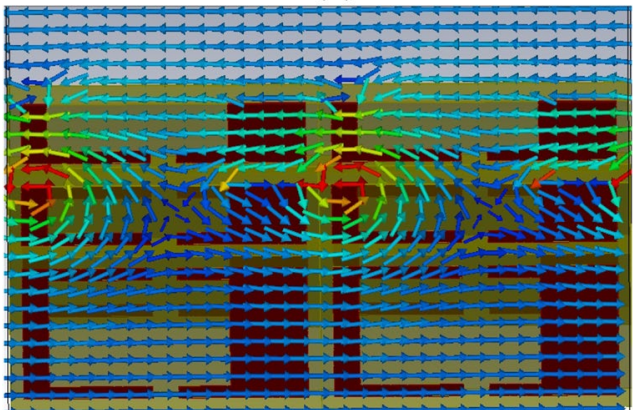

(b)
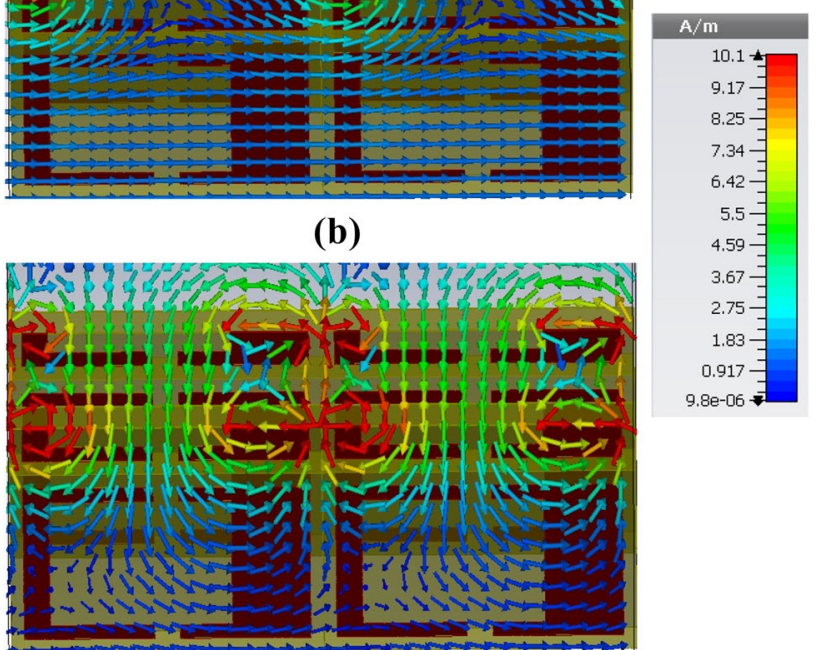

Fig. 6 Simulated magnetic field distributions at a $2.6 \mathrm{GHz}$ and b $2.92 \mathrm{GHz}$ resulting in a transmission tunnel band. The higher resonance at $2.87 \mathrm{GHz}$ shown in Fig. $6 \mathrm{~b}$ is found to be hybrid in nature. It is interesting to note that toroidal dipole moment $T_{y}$ is excited here by the longitudinal inter-layer coupling between the symmetric arms along the direction of propagation. The magnetic dipole moment $M_{z}$ excited on the consecutive arms will be in phase and is verified by the presence of a longitudinal component of magnetic fields in between the loops. The peculiarity of the design is that stacking results in the creation of an additional toroidal moment for the Fano resonance which significantly modifies the field pattern. The fields are distributed on both the substrate and metallic parts which gives a high field localization within the unit cell. The excitation of this toroidal moment significantly improves the quality factor of second resonance. The enhanced magnetic field circulation around the metallic parts indicates the presence of enhanced toroidal dipole moment for this resonance as compared to the first resonance. The magnetic field distribution is highly localized for the second resonance in comparison with that of the single layer asymmetric structure. The enhanced toroidal excitation for the second resonance significantly enhances the $\mathrm{Q}$-factor of the resonance as compared to the single layer Fano resonance structure. The presence of toroidal excitation is clearly seen in Fig. $5 \mathrm{~b}$ as longitudinal coupling which is usually absent in conventional metamaterials exhibiting Fano resonances [15]. This gives the opportunity for an additional degree of freedom for controlling electromagnetic wave propagation. In high index dielectric particle-based configuration [28]; only transverse coupling within a layer is used to create dual-band response. This is due to the fact that coupling is achieved by the virtue of displacement currents. This type of dual-band in-plane coupling is practically impossible for asymmetric metasurface containing metallic implants. This is because of the fact that the proposed metasurface, containing metallic implants as the unit cell, works on the basis of conduction current. In these structures, intra-layer coupling by implementing cascading is the only possibility for the creation of an additional resonant toroidal mode.

\section{Parametric analysis}

A detailed parametric analysis has been performed in CST Microwave Studio to find out the effect of different structural parameters on metamaterial resonances. Figure 7 illustrates the effect of loading height $h_{1}$ in the transmission coefficient of the structure for normal incidence. It is observed that for a lower loading height $\left(h_{1}=0.5 \mathrm{~mm}\right)$, a single resonant transmission peak of $2.7 \mathrm{GHz}$ is observed which is due to the combined effect of toroidal, electric and magnetic dipoles. Increasing loading height splits this 


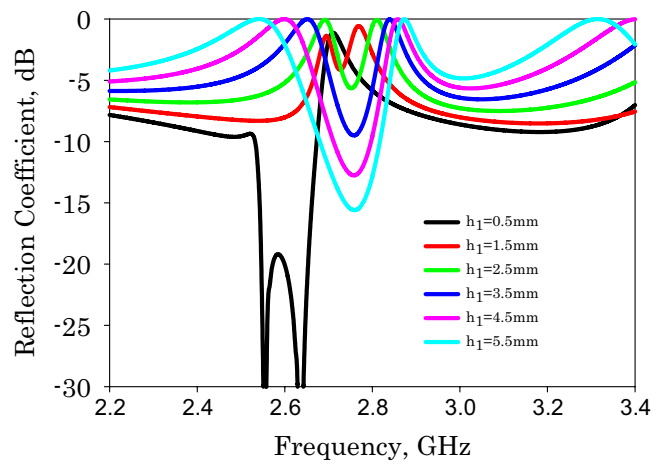

Fig. 7 Effect of stacking height on transmission characteristics

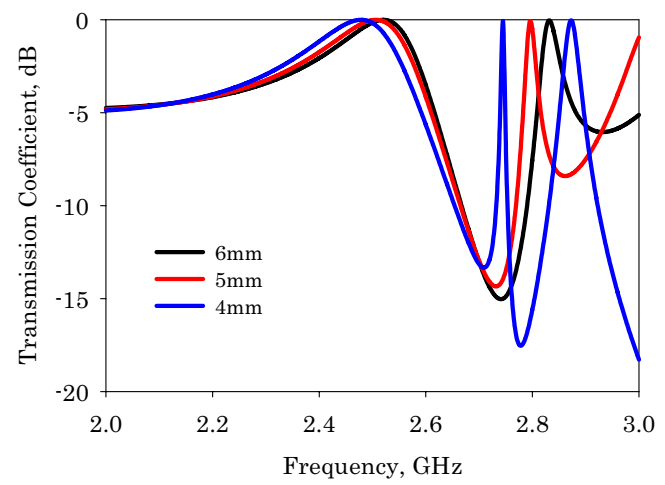

Fig. 8 Effect of asymmetry on transmission coefficient

resonance into two, and as the loading height is increased, the two resonances are found to shift toward opposite sides. The lower shifted resonance is identified as the toroidal resonance, and the higher shifted one is identified as the hybrid resonance of the composite. These conclusions are finalized from the multipolar scattering studies, and the results of such studies are omitted here for brevity.

We have also studied the effect of asymmetry on the transmission coefficient of the composite, and these results are illustrated in Fig. 8. Here the horizontal parameter of the asymmetric arm is varied in simulations. It is seen that decreasing the value of horizontal metalization slightly shifts the toroidal resonance to higher frequencies. But this variation is negligibly small. A significant lower shift is observed for the hybrid resonance. Higher asymmetry results in lower quality factor for the hybrid resonance.

\section{Conclusions}

An experimental realization of toroidal metamaterial in the microwave S-band frequencies is proposed in this study. The toroidal metamaterial constitutes a stacked asymmetric double split-ring resonator array printed on a low-cost epoxy substrate. It is worth mentioning that cascading the metasurface results in both transverse and longitudinal magnetic couplings resulting in dual-band toroidal response. It is noted that the presence of toroidal moment significantly enhances the quality factor of the Fano resonant transmission peak. The stacking thickness plays a crucial role behind the spectral separation and is optimized using computer simulations. Magnetic field map and multipole scattering theory reveal the excitation of dual-band toroidal moments in the composite under plane wave incidence.

Funding The authors acknowledge the financial support received from Department of Science and Technology (DST), Government of India, for the Project ECR/2017/002204.

\section{Compliance with ethical standards}

Conflict of interest The authors declare that they have no conflicts of interest.

\section{References}

1. Jackson JD (1999) Classical electrodynamics, 3rd edn. Wiley, Hoboken

2. Zhu BO, Zhao J, Feng Y (2013) Active impedance metasurface with full 3600 reflection phase tuning. Nat Sci Rep 49:1-6

3. Zhu BO, Chen K, Jia N, Sun L, Zhao J, Jiang T, Feng Y (2014) Dynamic control of electromagnetic wave propagation with the equivalent principle inspired tunable metasurface. Nat Sci Rep 4:1-7

4. Zel'dovich IB (1958) The relation between decay asymmetry and dipole moment of elementary particles. Sov Phys JETP 6:1184

5. Afanasiev GN, Stepanovsky YP (1995) The electromagnetic field of elementary time-dependent toroidal sources. J Phys A Math Gen 28:4565

6. Guo L-Y, Li M-H, Huang X-J, Yang H-L (2014) Electric toroidal metamaterial for resonant transparency and circular crosspolarization conversion. Appl Phys Lett 105:033507

7. Fedotov VA, Rogacheva AV, Savinov V, Tsai DP, Zheludev NI (2013) Resonant transparency and non-trivial non-radiating excitations in toroidal metamaterials. Nat Sci Rep 3:2967

8. Marinov K, Boardman AD, Fedotov VA, Zheludev N (2007) Toroidal metamaterial. New J Phys 9:1-12

9. Kaelberer T, Fedotov VA, Papasimakis N, Tsai DP, Zheludev NI (2010) Toroidal dipolar response in a metamaterial. Science 330:1510-1512

10. Gupta $M$, Singh R (2016) Toroidal versus Fano resonances in high Q planar THz metamaterials. Adv Opt Mater 201600553:1-7

11. Gupta M, Savinov V, Xu N, Cong L, Dayal G, Shuang W, Zhang W, Zheludev NI, Singh R (2016) Sharp toroidal resonances in planar terahertz metasurfaces. Adv Mater 201601611:1-6

12. Han S, Cong L, Gao F, Singh R, Yang H (2016) Observation of Fano resonance and classical analog of electromagnetically induced transparency in toroidal metamaterials. Ann Phys (Berl) 528(352-357):2

13. Gupta M, Srivastava YK, Manjappa M, Singh R (2017) Sensing with toroidal metamaterial. Appl Phys Lett 110:121108

\section{SN Applied Sciences}


14. Gupta M, Srivastava YK, Singh R (2017) A toroidal metamaterial switch. Adv Mater 30:201704845

15. Fan Y, Wei Z, Li H, Chen H, Soukoulis CM (2013) Low-loss and high-Q planar metamaterial with toroidal moment. Phys Rev $B$ $87: 115417$

16. Kim S-H, Oh SS, Kim K-J, Kim J-E, Park HY, Hess O, Kee C-S (2015) Sub-wavelength localization and toroidal dipole moment of spoof surface plasmon polaritons. Phys Rev B 91:035116

17. Tasolamprou AC, Tsilipakos O, Kafesaki M, Soukoulis CM, Economou EN (2016) Toroidal eigenmodes in all-dielectric metamolecules. Phys Rev B 94:205433

18. Dong Z-G, Ni P, Zhu J, Yin X, Zhang X (2012) Toroidal dipole response in a multifold double ring metamaterial. Opt Exp 20:13065

19. Dong Z-G, Zhu J, Rho J, Li J-Q, Lu C, Yin X, Zhang X (2012) Optical toroidal dipolar response by an asymmetric double-bar metamaterial. Appl Phys Lett 101:144105

20. Dong Z-G, Zhu J, Yin X, Li J, Lu C, Zhang X (2013) All-optical Hall effect by the dynamic toroidal moment in a cavity-based metamaterial. Phys Rev B 87:245429

21. Papasimakis N, Fedotov VA, Savinov V, Raybould TA, Zheludev NI (2016) Electromagnetic toroidal excitations in matter and free space. Nat Mater 15:263-271

22. Miroshnichenko AE, Evlyukhin AB, Yu YF, Bakker RM, Chipouline A, Kuznetsov Al, Luk'yanchuk B, Chichkov BN, Kivshar YS (2015)
Nonradiating anapole modes in dielectric nanoparticles. Nat Commun 6:8069

23. Nemkov NA, Stenishchev IV, Basharin AA (2017) Nontrivial nonradiating all-dielectric anapole. Nat Sci Rep 7:1064

24. Basharin AA, Chuguevsky V, Volsky N, Kafesaki M, Economou EN (2017) Extremely high Q-factor metamaterials due to anapole excitation. Phys Rev B 95:035104

25. Wu PC, Liao CY, Savinov V, Chung TL, Chen WT, Huang Y-W, Wu PR, Chen Y-H, Liu A-Q, Zheludev NI, Tsai DP (2018) Optical anapole metamaterial. ACS Nano 12:1920-1927

26. Ospanova AK, Labate G, Matekovits L, Basharin AA (2018) Multipolar passive cloaking by nonradiating anapole excitation. Nat Sci Rep 8:12514

27. Stenishchev IV, Basharin AA (2017) Toroidal response in alldielectric metamaterials based on water. Nat Sci Rep 7:9468

28. Su X, Sayanskiy A, Kupriianov AS, Tuz VR, Kapitanova P, Sun H-B, Han W, Kivshar YS (2018) Experimental observation of toroidal dipole modes in all-dielectric metasurface. Adv Opt Mater 801166:1-9

Publisher's Note Springer Nature remains neutral with regard to jurisdictional claims in published maps and institutional affiliations. 